\section{Criptococosis cerebral, voriconazol, niveles plasmáticos y síndrome de reconstitución inmune. Reporte de un caso}

\author{
MARÍA TÉLLEZ R. ${ }^{a}$, CATALINA SALGUEIRO C. ${ }^{a}$, \\ MARCELO LEIVA HERNÁNDEZ ${ }^{1}$, ALBERTO FICA ${ }^{2}$
}

\section{Cerebral cryptococcosis and immune reconstitution inflammatory syndrome. Case report}

We report a 45-year-old male with AIDS who had a Cryptococcus neoformans central nervous system infection. He was treated with amphotericin $B$ deoxycholate subsequently changed to voriconazole due to systemic toxicity of the former. Plasma levels of voriconazole were insufficient with a standard dose $(0.7 \mu \mathrm{g} / \mathrm{mL})$, therefore, the dose was increased thereafter to reach appropriate levels $(4.5 \mu \mathrm{g} / \mathrm{mL})$. Anti-retroviral therapy was started five weeks after voriconazole initiation with non-interacting drugs and he was discharged after a favorable evolution. He was re-admitted three months later due to seizures; a brain magnetic resonance showed new sub-cortical nodules. After excluding alternative causes and demonstrating fungal eradication, an immune reconstitution inflammatory syndrome (IRIS) event was suspected and treated with a short course of steroids. His evolution was satisfactory.

(Rev Med Chile 2018; 146: 1481-1485)

Key words: Acquired Immunodeficiency Syndrome; Drug monitoring; Immune Reconstitution Inflammatory Syndrome; Meningitis, Cryptococcal; Voriconazole.

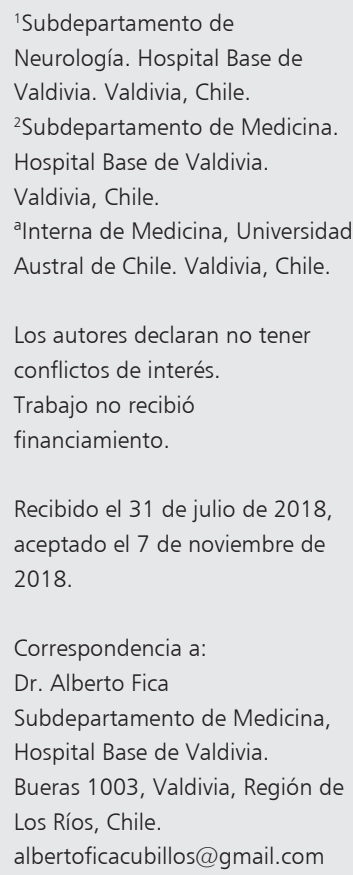

Los autores declaran no tener conflictos de interés.

Trabajo no recibió

financiamiento.

Recibido el 31 de julio de 2018 aceptado el 7 de noviembre de 2018.

Correspondencia a:

Dr. Alberto Fica

Subdepartamento de Medicina, Hospital Base de Valdivia.

Bueras 1003, Valdivia, Región de Los Ríos, Chile.

albertoficacubillos@gmail.com

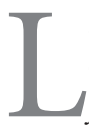

a criptococosis es una infección micótica oportunista causada por Cryptococcus neoformans, asociada a suelos contaminados con excrementos de palomas y otras aves. La infección se adquiere por inhalación de esporas con predilección por el tejido pulmonar y el sistema nervioso central (SNC) y contribuye a la mortalidad en pacientes con síndrome de inmunodeficiencia adquirida (SIDA) $)^{1-3}$. El compromiso del SNC incluye casos de meningitis o formas nodulares llamadas criptococomas ${ }^{1,2}$. Esta infección se expresa ante una inmunodepresión avanzada ${ }^{2,3}$.

El objetivo de esta comunicación es presentar un caso de criptococosis cerebral (CC) en un paciente con SIDA que debió ser tratado con voriconazol por intolerancia a la anfotericina, incluyendo la medición de sus niveles plasmáticos y que evolucionó con un síndrome inflamatorio de reconstitución inmune (SIRI). Tanto el tratamiento con voriconazol, la necesidad de medir sus niveles plasmáticos en pacientes con SIDA y el desarrollo de un SIRI bien documentado con exclusión de causas alternativas, representan aspectos novedosos en el manejo de pacientes con CC.

\section{Caso clínico}

Paciente masculino de 45 años que ingresó por 3 semanas de alteración conductual y de la marcha, 


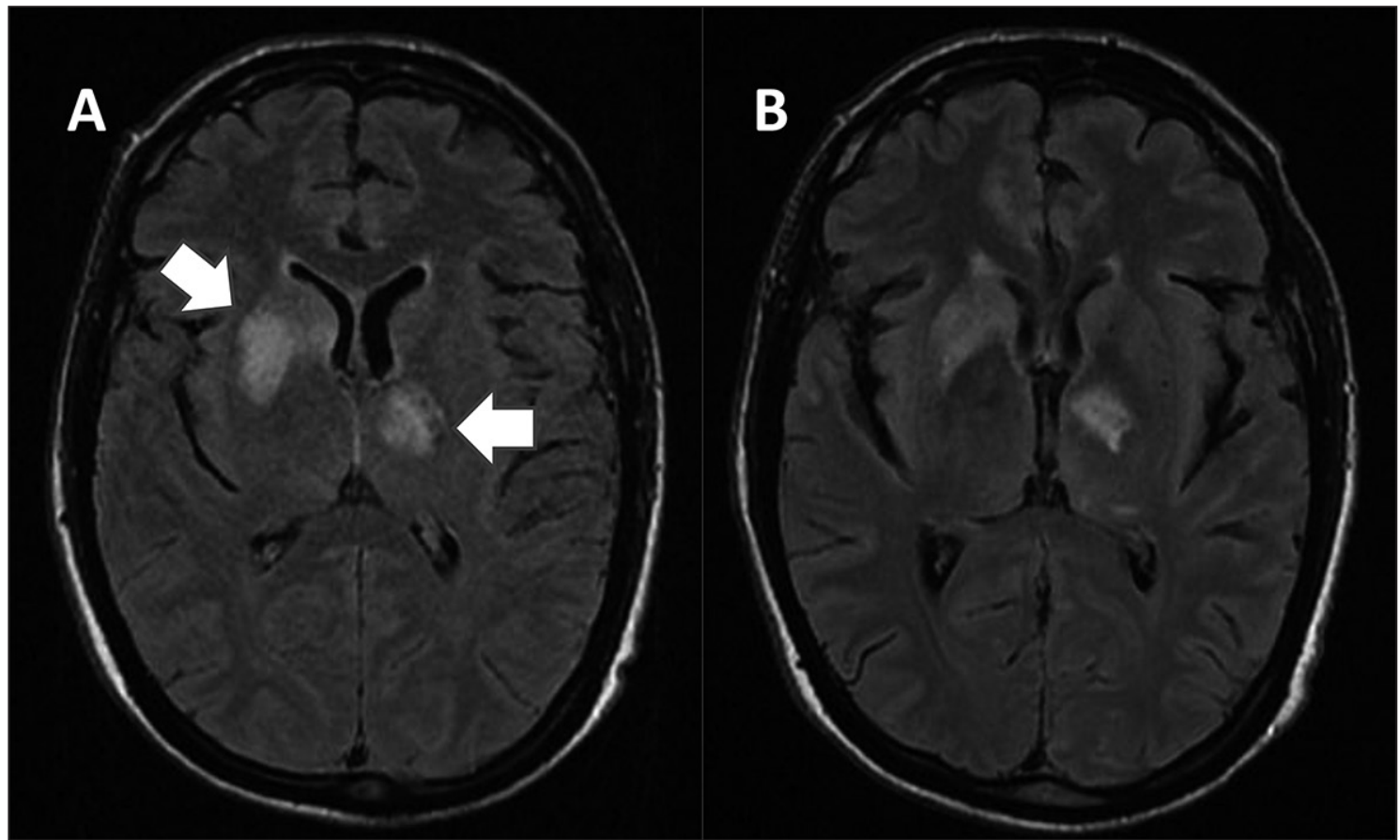

Figura 1. A: Imagen FLAIR (Fluid Attenuated Inversion Recovery) de RM cerebral al ingreso. Se aprecia compromiso de ganglios basales (flechas) con hiperintensidad por edema, que rodea las lesiones y que determina un efecto de masa sobre el ventrículo derecho. B: Control al mes de tratamiento que demuestra disminución del edema asociado a las lesiones. El paciente dio su consentimiento para utilizar estas imágenes y la información clínica asociada.

cefalea, incontinencia urinaria, candidiasis bucaly baja de peso. Presentaba bradipsiquia, alteración del lenguaje y focalización neurológica con reflejo corneal disminuido a derecha y paresia facio-braquio-crural derecha, sin signos meníngeos. En los exámenes destacaba anemia (hemoglobina $10 \mathrm{~g} / \mathrm{dL})$, linfopenia $(800 / \mu \mathrm{L})$, hipoalbuminemia $(3,4 \mathrm{~g} / \mathrm{dL})$, e hiponatremia (126 mEq/l). La resonancia magnética cerebral (RM) reveló lesiones hiperintensas en los ganglios basales con refuerzo (Figura 1A). Se documentó un test VIH positivo, un recuento CD4 de $25 / \mu \mathrm{L}$ y una carga viral $(\mathrm{CV})$ de 747.311 copias/mL. La infección por C. neoformans se confirmó por un test de tinta china y cultivo positivo en el líquido cefalorraquídeo (LCR), el que no demostró proteínas aumentadas (45 $\mathrm{mg} / \mathrm{dL}$ ) ni pleocitosis ( 0 células $/ \mathrm{mL}$ ). El paciente tenía contacto cotidiano con palomas. El estudio complementario del LCR no demostró bacterias con una RCP para T. gondii negativa. La serología para Trypanosoma cruzi, sífilis y T. gondii (IgG) fueron negativas.
Fue tratado con anfotericina B deoxicolato (anfoBdeox) pero presentó toxicidad con fiebre, temblores y calofríos que no mejoraron con infusión lenta, precarga salina y premedicación con hidrocortisona. Recibió alternativamente voriconazol ev con dosis de carga, dosis diaria ajustada a peso y cambio a vía oral a los 8 días (200 mg cada 12 h). La dosis tuvo que ser aumentada a $200 \mathrm{mg}$ cada $8 \mathrm{~h}$ por niveles plasmáticos valle insuficientes $(0,7$ $\mu \mathrm{g} / \mathrm{mL}$; deseable 1-5 $\mu \mathrm{g} / \mathrm{mL}$ ). Este ajuste permitió niveles plasmáticos valle adecuados $(4,5 \mu \mathrm{g} / \mathrm{mL})$. A las cinco semanas de tratamiento se inició terapia antirretroviral (TARV) con abacavir/lamivudina (HLA B5701 negativo) y raltegravir. Se constató mejoría neurológica completa y del estado general, lo que permitió su alta luego de 39 días con TARV, quimioprofilaxis con azitromicina y cotrimoxazol forte. El tratamiento con voriconazol fue cambiado a terapia de mantención con fluconazol $300 \mathrm{mg}$ /día oral luego de ocho semanas. LA RM reveló regresión parcial de las lesiones (Figura 1B). El control a las 11 semanas de TARV demostró un descenso 


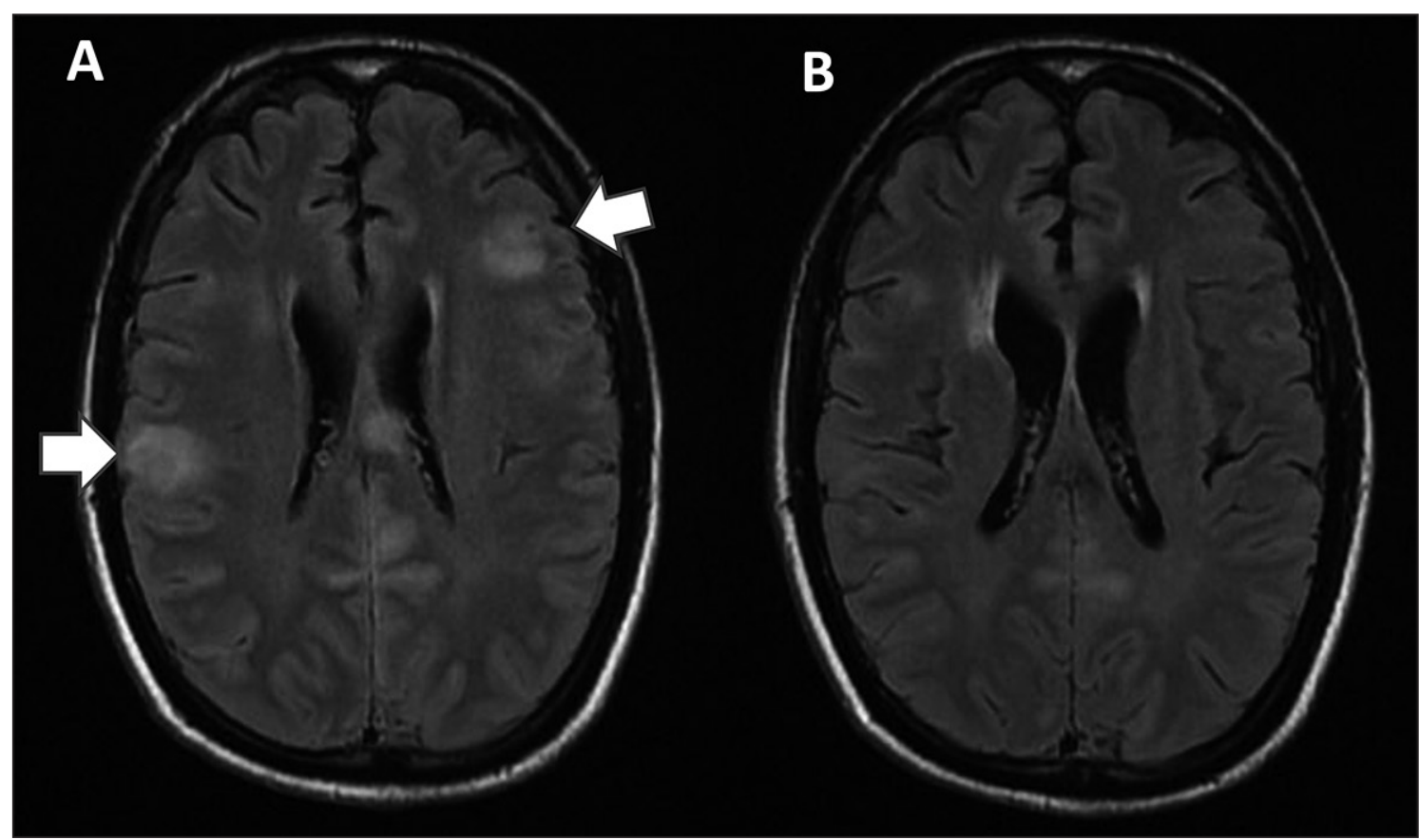

Figura 2. A: Imagen FLAIR (Fluid Attenuated Inversion Recovery) de RM cerebral al momento del reingreso. Se aprecian lesiones nodulares hiperintensas córtico-subcorticales en ambos hemisferios no presentes en estudios previos (Flechas). B: Control a las 3 semanas de tratamiento con corticoides que demuestra regresión de lesiones centrales. El paciente dio su consentimiento para utilizar estas imágenes y la información clínica asociada.

de la carga viral a 61.683 copias/mL y un aumento del recuento CD4 a 110/ $\mu \mathrm{L}$.

A los tres meses, el paciente reingresó por síndrome convulsivo sin signos de irritación meníngea, fiebre o focalización neurológica. La RM cerebral indicó nuevos nódulos subcorticales bilaterales hiperintensos (Figura 2A) y el estudio del LCR mostró proteínorraquia de $240 \mathrm{mg} / \mathrm{dL}$ con celularidad normal ( $3 / \mathrm{mL})$. El cultivo del LCR descartó nuevos agentes infecciosos y demostró la erradicación de C. neoformans. La RCP para tuberculosis resultó negativa, al igual que el estudio de T. gondii, virus de la familia herpes y virus JC. Se interpretó el cuadro convulsivo como SIRI por C. neoformans y se trató con dexametasona por cuatro días (10 mg cada $6 \mathrm{~h} \mathrm{ev}$ ), además de terapia anticonvulsivante con levetiracetam, para evitar las interacciones farmacológicas. La RM de control a las dos semanas demostró regresión casi total de las lesiones (Figura 2B). El paciente fue dado de alta con TARV, fluconazol y profilaxis con cotrimoxazol y sin corticoides.

\section{Discusión}

Nuestro caso de SIRI está convenientemente respaldado por el deterioro paradójico que tuvo el paciente luego de una mejoría clínica e imagenológica ante la infección inicial, por la reconstitución paralela de la inmunidad celular CD4, la aparición de nuevas lesiones, la erradicación del agente al presentarse la reacción paradójica, la exclusión de otras etiologías alternativas, la temporalidad compatible y la respuesta clínica a los corticoides sin otras terapias ${ }^{3}$.

Para el diagnóstico de un SIRI se han postulado elementos mínimos que corresponden a: a) la asociación temporal entre el inicio de la TARV y posterior desarrollo de síntomas; b) una restauración inmune demostrada por disminución de la carga viral y aumento de linfocitos CD4+, y c) presencia de signos y síntomas clínicos consistentes con un proceso inflamatorio ${ }^{4}$.

Hasta un tercio de los pacientes con CC desarrollarán un SIRI, que ocurre en promedio a 
las 10 semanas luego del inicio de la TARV ${ }^{3}$. El diagnóstico diferencial es amplio e incluye otras infecciones tales como toxoplasmosis, enfermedad de Chagas, nocardiosis, leucoencefalopatía multifocal progresiva, tuberculosis y linfoma primario del SNC. Las manifestaciones pueden ser similares al cuadro inicial o con presentación de nuevos síntomas. La exclusión de otras causas requiere estudio de imágenes, estudio microbiológico del LCR con técnicas convencionales o moleculares y/o una biopsia cerebral ${ }^{5,6}$. Esta exclusión podría explicar por qué, a pesar de ser un fenómeno relativamente frecuente en pacientes sobrevivientes de $\mathrm{CC}^{1-3}$, los reportes nacionales sobre esta complicación sean inexistentes y la documentación de algunos casos latinoamericanos ha sido indirecta y más bien basada en una respuesta inespecífica al uso de corticoides ${ }^{7}$.

Los factores de riesgo más importantes para el desarrollo de un SIRI por C. neoformans están representados por una alta carga microbiana y nivel de inmunusupresión inicial ${ }^{8}$. La respuesta inmune limitada retrasa la erradicación microbiológica, permitiendo la persistencia de antígenos al momento de iniciar la TARV. El riesgo de SIRI es 7 veces superior en aquellos pacientes que simultáneamente se presentan con $<25$ células/ $\mathrm{mL}$ y $<50 \mathrm{mg} / \mathrm{dL}$ de proteínas en el LCR, tal como ocurrió en nuestro paciente ${ }^{9}$. La reconstitución inmune permite que se genere un aumento de citoquinas que explica el síndrome $e^{8,10}$. El conocimiento de la importancia de la respuesta inmune en la génesis del SIRI y su asociación con la baja capacidad inicial para erradicar el agente ha llevado a postergar el inicio de la TARV. En efecto, el inicio diferido hasta 5 semanas se asocia a una menor mortalidad respecto a su inicio precoz en pacientes con $\mathrm{CC}^{11}$.

El tratamiento de los casos de SIRI por $C$. neoformans incluye el control de la hipertensión endocraneana y su tratamiento farmacológico es anecdótico, sin datos disponibles de ensayos clínicos. Se prefiere el uso de corticoides cuando existe una condición grave o persistente. La suspensión del TARV es rara vez necesaria ${ }^{8}$.

Nuestro caso presenta 2 hechos distintivos que ameritan ser discutidos: la presentación de la CC como lesiones nodulares y la necesidad de utilizar una terapia alternativa por la toxicidad sistémica ante anfotericina. Debido al predominio de lesiones nodulares por $T$. gondii, se suele indicar terapia antitoxoplasma, lo que puede retrasar el reconocimiento de otras causas, recomendando que ante pruebas serológicas negativas para $T$. gondii, neuroimágenes atípicas o una respuesta clínica fallida ante el tratamiento empírico se amplíe el estudio. El rendimiento de neuroimágenes ante el diagnóstico histopatológico por biopsia o autopsia en lesiones focales, mostró una especificidad de $75 \%$ para la tomografía computada o la RM en el diagnóstico de toxoplasmosis cerebral, subrayando la importancia del estudio directo ${ }^{12}$. Un estudio de autopsias demostró que la infección oportunista más frecuente del SNC era la criptococosis, sin embargo, el diagnóstico clínico apropiado solo fue propuesto en $50 \%{ }^{13}$. Así, nuestro caso cuestiona el paradigma de asignar una lesión nodular a una causa específica.

El paciente mantuvo una intolerancia sistémica ante anfoBdeox, el compuesto recomendado en pacientes con $\mathrm{CC}^{2,14}$, a pesar de aplicar estrategias como la precarga salina, infusiones lentas y premedicación con corticoides ${ }^{15,16}$. Voriconazol ha sido utilizado exitosamente en estudios observacionales en pacientes con $\mathrm{CC}^{17}$, con una adecuada penetración al SNC, pero tiene interacciones citocromales y una farmacocinética no lineal y errática ${ }^{18}$. La monitorización de sus niveles plasmáticos se ha asociado a una menor tasa de efectos adversos y una mejor respuesta clínica en pacientes oncohematológicos cuando se obtienen concentraciones valle entre 1 y $5,5 \mu \mathrm{g} / \mathrm{mL}^{19}$.

Los aspectos farmacocinéticos del voriconazol en pacientes con VIH/SIDA han sido poco explorados $^{21}$. En nuestro caso, el paciente no estaba recibiendo fármacos que interactuarán con voriconazol y el TARV no incluyó drogas con interacción, subrayando la relevancia de una farmacocinética no predecible aun en ausencia de interacciones farmacológicas.

En conclusión, la CC cerebral puede presentarse como meningitis o nódulos con un amplio diagnóstico diferencial. Ante toxicidad por anfoBdeox es necesario usar terapias alternativas como voriconazol, un compuesto activo sobre C. neoformans, con buena penetración al SNC y efectividad demostrada en estudios observacionales. Su farmacocinética errática hace necesaria la medición de sus niveles plasmáticos para asegurar un rango terapéutico y su seguridad, incluso en ausencia de interacciones citocromales. El SIRI es frecuente de observar en infecciones centrales por 
C. neoformans, existiendo criterios para su reconocimiento. Las opciones de manejo son diversas, incluyendo el uso de corticoides.

\section{Referencias}

1. Chastain DB, Henao-Martínez AF, Franco-Paredes C. Opportunistic invasive mycoses in AIDS: cryptococcosis, histoplasmosis, coccidiodomycosis, and talaromycosis. Curr Infect Dis Rep 2017; 19 (10): 36.

2. Bicanic T, Harrison TS. Cryptococcal meningitis. Br Med Bull 2004; 72: 99-118.

3. da Cunha Colombo ER, Mora DJ, Silva-Vergara ML. Immune reconstitution inflammatory syndrome (IRIS) associated with Cryptococcus neoformans infection in AIDS patients. Mycoses 2011; 54 (4): e178-82.

4. Hidrón A, González A. Síndrome inflamatorio de reconstitución inmune en pacientes infectados con el virus de la inmunodeficiencia humana y afecciones fúngicas. Infectio 2012; 16 (Supl 3): 51-8.

5. Miranda G, Díaz C, Delllen H, Hermosilla H. Enfrentamiento imaginologico de las lesiones cerebrales en pacientes VIH. Rev Chil Radiol 2008;14 (4): 200-207.

6. Fica A, Salinas M, Jercic MI, Dabanch J, Soto A, Quintanilla S, et al. Enfermedad de Chagas del sistema nervioso central en un paciente con SIDA demostrada por métodos cuantitativos moleculares. Rev Chilena Infectol 2017; 34 (1): 69-76.

7. Guevara-Silva EA, Ramírez-Crescencio MA, Soto-Hernández JL, Cárdenas G. Central nervous system immune reconstitution inflammatory syndrome in AIDS: experience of a Mexican neurological centre. Clin Neurol NeuroSurg 2012; 114 (7): 852-61.

8. Bahr N, Boulware DR, Marais S, Scriven J, Wilkinson RJ, Meintjes G, et al. Central nervous system immune inflammatory reconstitution syndrome. Curr Infect Dis Rep 2013; 15 (6): 583-93.

9. Boulware DR, Bonham SC, Meya DB, Wiesner DL, Park GS, Kambugu A, et al. Paucity of of initial cerebrospinal fluid inflammation in cryptococcal meningitis is associated with subsequent immune reconstitution inflammatory syndrome. J Infect Dis 2010; 202 (6): 962-70.

10. Wiesner DL, Boulware DR. Cryptococcus-related immune reconstitution inflammatory Syndrome (IRIS): Pathogenesis and its clinical implications. Curr Fungal Infect Rep 2011; 5 (4): 252-61.

11. Boulware DR, Meya DB, Muzoora C, Rolfes MA, Hu- ppler Hullsiek K, Musubire A, et al. Timing of antiretroviral therapy after diagnosis of cryptococcal meningitis. N Engl J Med 2014; 370 (26): 2487-98.

12. Shyam Babu C, Satishchandra P, Mahadevan A, Pillai Shibu V, Ravishankar S, Sidappa N, et al. Usefulness of stereotactic biopsy and neuroimaging in management of HIV-1 clade C associated focal brain lesions with special focus on cerebral toxoplasmosis. Clin Neurol Neurosurg 2013; 115 (7): 995-1002.

13. Diaconu IA, Stratan LM, Nichita L, Aramă V, Moroti Constantinescu VR, Diaconu AI, et al. Diagnosing HIV-associated cerebral diseases-The importance of neuropathology in understanding HIV. Rom J Morphol Embryol 2016; 57 (2 Suppl): 745-50.

14. Perfect JR, Dismukes WE, Dromer F, Goldman DL, Graybill JR, Hamill RJ, et al. Clinical practice guidelines for the management of cryptococcal disease: 2010 update by the Infectious Diseases Society of America. Clin Infect Dis 2010; 50 (3): 291-322.

15. Branch RA. Prevention of amphotericin B-induced renal impairment. Arch Intern Med 1988; 148 (11): 2389-94.

16. Eriksson U, Seifert B, Schaffner A. Comparison of effects of amphotericin B deoxycholate infused over 4 hours or 24 hours: randomized controlled trial. Br Med J 2001; 322 (7286): 1-6.

17. Yao Y, Zhang JT, Yan B, Gao T, Xing XW, Tian CL, et al. Voriconazole: a novel treatment option for cryptococcal meningitis. Infect Dis (Lond) 2015; 47 (10): 694-700.

18. Pascual A, Nieth V, Calandra T, Bille J, Bolay S, Decosterd LA, et al. Variability of voriconazole plasma levels measured by new high-performance liquid chromatography and bioassay methods. Antimicrob Agents Chemother 2007; 51 (1): 137-43.

19. Park WB, Kim NH, Kim KH, Lee SH, Nam WS, Yoon $\mathrm{SH}$, et al. The effect of therapeutic drug monitoring on safety and efficacy of voriconazole in invasive fungal infections: a randomized controlled trial. Clin Infect Dis 2012; 55 (8):1080-7.

20. Nierenberg NE, Thompson GR, Lewis JS, Hogan BK, Patterson TF. Voriconazole use and pharmacokinetics in combination with interferon-gamma for refractory cryptococcal meningitis in a patient receiving low-dose ritonavir. Med Mycol 2010; 48 (3): 532-6.

21. Carbonara S, Regazzi M, Ciracì E, Villani P, Stano $\mathrm{F}$, Cusato $\mathrm{M}$, et al. Long-term efficacy and safety of TDM-assisted combination of voriconazole plus efavirenz in an AIDS patient with cryptococcosis and liver cirrhosis. Ann Pharmacother 2009; 43 (5): 978-84. 